\title{
SAMMLUNG ME'TZLER
}

M 1 Raabe Einfübrung in die Bücherkunde

M 3 Meisen Altdeutsche Grammatik II: Formenlebre

M 4 Grimm Bertolt Brecht

M 5 Moser Annalen der deutschen Sprache

M 6 Schlawe Literarische Zeitschriften 1885-1910

M 7 Weber/Hoffmann Nibelungenlied

M 8 Meyer Eduard Mörike

M 9 Rosenfeld Legende

M 10 Singer Der galante Roman

M 12 Nagel Meistersang

M 13 Bangen Die schriftliche Form germanist. Arbeiten

M 14 Eis Mittelalterliche Fachliteratur

M 15 Weber/Hoffmann Gottfried von Straßburg

M 16 Lüthi Märchen

M 17 Wapnewski Hartmann von Aue

M 18 Meetz Friedrich Hebbel

M 19 Schröder Spielmannsepik

M 20 Ryan Friedrich Hölderlin

M 22 Danzel Zur Literatur und Pbilosopbie der Goethezeit

M 24 Schlawe Literarische Zeitschriften 1910-1933

M 25 Anger Literarisches Rokoko

M 26 Wodtke Gottfried Benn

M 27 von Wiese Novelle

M 28 Frenzel Stoff-, Motiv- und Symbolforschung

M 29 Rotermund Christian Hofmann von Hofmannswaldau

M 30 Galley Heinrich Heine

M 31 Müller Franz Grillparzer

M 32 Wisniewski Kudrun

M 33 Soeteman Deutsche geistliche Dichtung des 11.u. 12. Jh.s

M 34 Taylor Melodien des Mittelalters I: Darstellung

M 35 Taylor Melodien des Mittelalters II: Materialien

M 36 Bumke Wolfram von Eschenbach

M 37 Engel Handlung, Gespräch u. Erzäblung. Faksimiledruck

M 38 Brogsitter Artusepik

M 39 Blanckenburg Versuch über den Roman. Faksimiledruck

M 40 Halbach Walther von der Vogelweide

M 41 Hermand Literaturwissenschaft und Kunstwissenschaft

M 43 Glinz Deutsche Syntax 


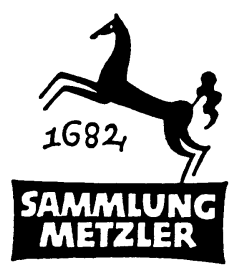

REALIEN ZUR LITERATUR

ABT. D:

LITERATURGESCHICHTE 


\author{
HELMUT DINSE \\ SOL LIPTZIN
}

\title{
Einführung
}

in die

\section{jiddische Literatur}


Die Verfasser danken Dagmar Hoffmann-Fracchetti für ihre Mitarbeit.

CIP-Kurztitelaufnahme der Deutschen Bibliothek Dinse, Helmut

Einführung in die jiddische Literatur / Helmut Dinse; Sol Liptzin. - 1. Aufl. - Stuttgart:

Metzler, 1978.

(Sammlung Metzler: M 165: Abt. D, Literaturgeschichte)

ISBN 978-3-476-10165-5

NE: Liptzin, Sol:

ISBN 978-3-476-10165-5

ISBN 978-3-476-03871-5 (eBook)

DOI 10.1007/978-3-476-03871-5

\section{165}

(C) Springer-Verlag GmbH Deutschland 1978

Ursprünglich erschienen bei J. B. Metzlersche Verlagsbuchhandlung und Carl Ernst Poeschel Verlag GmbH in Stuttgart 1978 
Vorwort . . . . . . . . . . . . . VII

Bibliographische Vorbemerkungen . . . . . . . IX

Teil I

Die ältere jiddische Literatur bis zur Aufklärung (Helmut Dinse)

I. Die Anfänge der jiddischen Literatur . . . . . . 1

1. Erste schriftliche Spuren jiddischer Sprache . . 1

2. Frühe religiöse Gebrauchsliteratur . . . . . . 3

a) Glossen und Glossare . . . . . . . . . . 3

b) Bibelkommentare und Bibelübersetzungen . 5

c) Gebetbücher . . . . . . . . . . . . . 8

d) Minhagim . . . . . . . . . . . . . . . . 9

3. Frühe weltliche Sachliteratur . . . . . 11

a) Privatbriefe . . . . . . . . . . . . 11

b) Juristische Schriftstücke . . . . . . . . . 12

c) Kalender und Merkbücher . . . . . . . . 14

4. Weltliche und geistliche Dichtung des Mittelalters . . . . . . . . . . . . . 14
a) Jüdische Folklore
b) Spielmannsdichtung . . . . . . . . . . 17
c) Geistliche Epik . . . . . . . . . . . . 22
d) Erzählungen . . . . . . . . . . . 26

II. Die jiddische Literatur im 16.-17. Jahrhundert . . 28

1. Erste Druckwerke . . . . . . . . . . . 28

2. Frauenliteratur . . . . . . . . 31

a) Biblische Literatur . . . . . . . . . 31

b) Liturgische und rituelle Frauenbücher . . . 37

c) Frühe Mussarliteratur . . . . . . . . . 40

d) Spruchbücher . . . . . . . . . . . . . . 43

3. Die bürgerliche Literatur . . . . . . . . 45

a) Geschichtliche Werke . . . . . . . . 45

b) Jiddische Volksbücher . . . . . . . . 46

c) Religiöse Unterhaltungsliteratur . . . . 50

d) Dramatische Dichtung . . . . . . . . 55

4. Die kritische Mussarliteratur . . . . . . 57

a) Sittenspiegel . . . . . . . . . . 57 
b) Sozialkritische Mussarliteratur

c) Jiddische Literatur zum Gesellschaftsleben in Deutschland und Osteuropa im 17. Jahrhundert . . . . . . . . . . . .

d) Lyrische Mussardichtung und historisches Liedgut

III. Die jiddische Literatur im 17.-18. Jahrhundert . . 67

1. Bibelübersetzungen . . . . . . . . . 67

2. Ritualbücher . . . . . . . . . . 69

3. Gemeindeverordnungen (Takanos) . . . . . 71

4. Erbauungs- und Unterhaltungsliteratur . . . . 72

IV. Das Ende der älteren jiddischen Literatur . . . . 76

\section{Teil II}

Die neuere jiddische Literatur

(Sol Liptzin)

I. Der Neubeginn . . . . . . . . . . . . . . . 79

II. Der Aufstieg . . . . . . . . . . . . . . . . 84

III. Der klassische Höhepunkt . . . . . . . . . . 91

IV. Die jiddische Literatur zwischen den beiden Weltkriegen . . . . . . . . . . . . . 104

1. Sowjetrußland . . . . . . . . . . 104

2. Polen . . . . . . . . . . . . . 110

3. Rumänien . . . . . . . . . . . . 118

4. Westeuropa . . . . . . . . . . 121

V. Jiddische Literatur in Amerika . . . . . . . . 124

VI. Die jiddische Literatur während der Zeit des Nationalsozialismus . . . . . . . . . . 138

VII. Spätstadium und weltweite Ausbreitung der jiddischen Literatur . . . . . . . . . . 145

VIII. Die jiddische Literatur in Israel . . . . . . 163

Register . . . . . . . . . . . . 173 
Die jiddische Literatur ist das Ausdrucksmittel des aschkenasischen Judentums, das am Vorabend des Zweiten Weltkrieges durch etwa zehn Millionen Juden, vornehmlich ost- und mitteleuropäischer Provenienz, repräsentiert wurde. Die in ihr zum Ausdruck kommende jiddische Sprache, die als jüdische Verkehrssprache ihren Ursprung im frühen Mittelalter hat, vereint in sich mehrere Komponenten: aufbauend auf den älteren Schichten des Hebräischen und Aramäischen und bereichert durch griechische, lateinische und altromanische Elemente, bildet das Mittelhochdeutsche den Hauptbestandteil des Jiddischen, berücksichtigt man seine grammatische Grundstruktur und seinen Wortschatz, der zu mehr als vier Fünfteln deutscher Herkunft ist. Jüdische Einwanderer, die während der Karolingerzeit, aus Frankreich und Nord-Italien einwandernd, deutsches Sprachgebiet betraten und sich an Rhein, Mosel, Main und Donau niederließen, nahmen im Umgang mit ihren nicht-jüdischen Nachbarn deutsche Ausdrücke und Redewendungen in ihre Muttersprache auf, um sie so mit den älteren hebräischen, aramäischen, alt-französischen und alt-italienischen Elementen $\mathrm{zu}$ vermischen. Gezwungen durch die Pogrome im Zuge des ersten Kreuzzuges (1096) und die sich anschließenden Verfolgungen im gesamten mitteleuropäischen Raum, die im Judenmassaker des Jahres 1348 ihren Höhepunkt fanden, flohen jüdische Familien in Massen nach Osten, wo sie sich in den Ländern des slawischen Raumes niederließen. Hier erfolgte eine weitere sprachliche Assimilation, indem etliche Slawismen in die jiddische Sprache einflossen. So bildete sich ein grundlegender Unterschied $z$ wischen dem Jiddisch dieser nunmehr an der Oder, im Baltikum, am Schwarzen Meer oder am Dnjepr beheimateten Juden und dem Jiddisch jener in Mitteleuropa verbliebenen Juden heraus, der auch nicht durch die im 17. Jh. aus dem Ostraum zurückwandernden Jiddischsprecher egalisiert worden ist. Vielmehr entwickelten sich zwei jiddische Hauptidiome: das West-Jiddisch und das Ost-Jiddisch. Die geistigen Strömungen der Aufklärung und die Emanzipationsbestrebungen Moses Mendelssohns führten im 18. Jh. dazu, daß dem West-Jiddisch nur noch wenig Überlebensraum gegeben war, während das Ost-Jiddisch seine Blüte und Ausbreitung durch die Herausbildung und Entwicklung einer neuzeitlichen Literatur erst erreichen sollte. 
Der erste Teil der vorliegenden $»$ Einführung in die jiddische Literatur « ist der älteren jiddischen Literatur gewidmet, die zwischen dem 13. und 18. Jh. erblühte, als das West-Jiddische dominierte. Der zweite Teil macht den Leser mit der neueren jiddischen Literatur vertraut, die in der Verselbständigung des Ost-Jiddischen im 19. Jh. ihre Geburt erlebte. Die durch das 1925 gegründete Jiddische Wissenschaftliche Institut (YIVO) vorgenommene Standardisierung der jiddischen Sprache ermöglichte den Eintritt der jiddischen Literatur in die Hauptströme der Weltliteratur, und die Erforschung der jiddischen Sprache und Literatur hat längst einen gesicherten Platz an europäischen, amerikanischen und israelischen Universitäten gewonnen. 


\section{BibLIOGRAPHISCHE VORBEMERKUNGEN}

Wegen der Begrenztheit des Raumes müssen die bibliographischen Angaben auf wenige Quellen beschränkt bleiben. Reichlicher sind wissenschaftliche Arbeiten über die ältere jiddische Literatur im Literaturverzeichnis zu Helmut Dinse, "Die Entwicklung des jiddischen Schrifttums im deutschen Sprachgebiet «, Stuttgart 1974 angeführt, worauf sich auch die Quellennummern im ersten Teil dieser Einführung beziehen. Die umfassendste bibliographische Quelle für die jiddische Literatur des 19. und 20. Jh.s ist das "Lexikon fun der jidischer Literatur», 8 Bde., New York $1956 \mathrm{ff}$. Von gleichem Wert dürfte Salman Reisens "Lexikon fun der jidischer Literatur", 4 Bde., Wilna 1928 sein, in dem sich zahlreiche kritische Anmerkungen $\mathrm{zu}$ einzelnen Autoren finden lassen. Zalman Zylbercweigs "Lexikon fun jidischen Teater ", 5 Bde., New York 1931-1967 ergänzt die beiden Literaturlexika durch umfassende Angaben zum jiddischen Theater und seinen Dramatikern und Schauspielern. (Diese drei Lexika sind in jiddischer Sprache verfaßt!) Die Encyclopedia Judaica, 16 Bde., Jerusalem 1972, Suppl. $1973 \mathrm{ff}$. (in Englisch!) bringt am Schluß jedes Artikels über einzelne jiddische Schriftsteller eine auf die wichtigsten Aufsätze und Darstellungen begrenzte bibliographische Auswahl.

Uberblicke über die jiddische Literatur finden sich in allen größeren Allgemeinenzyklopädien, besonders aber in jüdischen. Neben den grundlegenden älteren Arbeiten zur jiddischen Literatur (Max Erik, Di Geschichte fun der jidischer Literatur, Warschau 1928; jidd.; Leo Wiener, The History of Yiddish Literature in the 19th Century, New York 1899; Meyer Isser Pines, Histoire de la littérature judéo-allemande, Paris 1911 deutsche Ausg. Leipzig 1913) sind als geläufigere Darstellungen C. A. Madison, Yiddish Literature, 1968 und Sol Liptzin, A History of Yiddish Literature, 1972 zu nennen.

Eine Bibliographie der jiddischen Literatur in englischer Úbersetzung, die D. Abramovich vorbereitete, gab YIVO 1967 heraus. Sie wird jährlich ergänzt. Im >Jewish Book Annuak, ebenfalls von D. Abramovich vorbereitet und vom Jewish Book Council of America herausgegeben, werden alle Bibliographien jiddischer Buchveröffentlichungen auf der ganzen Welt erfaßt. Diese Bibliographie der Bibliographien erschien 1978 in ihrem 35. Band.

Anthologien jiddischer Literatur liegen in Max Grünbaums 
"Jüdischdeutscher Chrestomathie" (Leipzig 1882) sowie in englischer Ubersetzung I. Howes und E. Greenbergs "Treasury of Yiddish Poetry " (1972), "Treasury of Yiddish Stories « (1972) und "Voices from the Yiddish « (1972) wie auch in J. Landis "The Great Yiddish Plays (1972) und J. Leftwich »Anthologie of Modern Yiddish Literature« (1974) vor.

Ins deutsche Schriftbild übertragene jiddische Texte finden sich in: Otto F. Best, Mameloschen. Jiddisch - Eine Sprache und ihre Literatur, Frankfurt/M. 1973 sowie in Siegmund A. Wolf, Jiddisches Wörterbuch, Mannheim 1962, dessen Einleitung ebenso wie Salcia Landmann, Jiddisch. Abenteuer einer Sprache, Olten-Freiburg i. Br. 1962 wesentliche Grundzüge der jiddischen Sprachgeschichte vermittelt. Eine Sammlung jiddischer Erzählungen in deutscher Schreibweise bietet Immanuel Olsvanger, Rosinkess mit Mandeln, Basel 11920, ${ }^{21931 . ~ J i d d i s c h e ~ L i e d e r ~}$ mit deutscher Übersetzung und Notenanhang haben Lin Jaldati und Eberhard Rebling herausgegeben: Es brennt, Brüder, es brennt, (Ost) Berlin 11966, ${ }^{2} 1969$.

Deutsche Ausgaben moderner jiddischer Erzähler liegen u. a. vor in: Schalom Asch, Der Apostel, 1946; Der Nazarener, 1950; Maria, 1950; David Bergelson, Ausgewählte Werke, 4 Bde., 1922; Das Ende vom Lied, 1965; Eisik Meir Dick, Ausgewählte Werke, 2 Bde., 1922; dass. in einem Band, 1954; Itzik Manger, Das Buch vom Paradies, 1963; Mendele Mocher Sforim, Werke, 2 Bde., 1961/62; Jitzchok Leibusch Peretz, Jüdische Geschichten, 1916; Chassidische Geschichten, 1917; Aus dieser und jener Welt, 1919; Drei Dramen, 1920; Die Zeit, 1923; Erzählungen aus dem Ghetto, 1961; Der Golem, 1967; Baal Schem als Ehestifter u. a. Erzählungen, 1969; Scholem Aleichem, Stempenju, 11888, 21925; Aus dem Nahen Osten, 1914, 21922; Die Geschichte Tewjes des Milchhändlers ${ }^{1} 1914,{ }^{2} 1922$, Neuausgabe 1955 und 1960 unter dem Titel Tewje, der Milchmann; Die erste jüdische Republik, 11919, 21925; Menachem Mendel, 1921, Neuausgabe 1962; Eine Hochzeit ohne Musikanten, 1961; Der Sohn des Kantors, 1965, auch unter dem Titel Mottl, der Kantorssohn, 1965; Der behexte Schneider, 1969; Isaac Bashevis Singer, Jakob der Knecht, 1965; Der Zauberer von Lublin, 1967; Gimpel der Narr, 1968; Massel und Schlammassel oder Die Milch einer Löwin, 1969; Als Schlemihl nach Warschau ging u. a. Geschichten, 1970; Israel Joshua Singer, Josche Kalb, 1967. 